DEAL WATCH

\title{
Epizyme-Celgene deal signals interest in new class of epigenetic drugs
}

Epizyme and Celgene have announced a partnership on the discovery, development and commercialization of inhibitors of histone methyltransferases (HMTs), an emerging family of epigenetic drug targets, for genetically defined cancers. Celgene, which markets two of the four epigenetic drugs currently approved - the histone deacetylase (HDAC) inhibitor romidepsin (Istodax) and the DNA methyltransferase inhibitor azacitidine (Vidaza) - gains exclusive rights outside the United States for Epizyme's preclinical DOT1L HMT inhibitor programme against mixed lineage leukaemia (MLL). The deal, which involves an upfront payment of US\$90 million to Epizyme, also provides Celgene with options to license ex-US rights to other Epizyme HMT inhibitor programmes, through which Epizyme could earn up to $\$ 160$ million in milestone payments.

Aberrant epigenetic changes resulting in gene dysregulation, such as oncogene overexpression or tumour suppressor silencing, can cause cancer. Taiping Chen, Associate Professor at the MD Anderson Cancer Center, Texas, USA, explains that: "Unlike irreversible genetic mutations, epigenetic mutations are potentially reversible, which makes epigenetic therapy an attractive approach for cancer treatment. Histone-modifying enzymes, including HDACs and HMTs, have attracted much interest in the context of anticancer drugs in recent years and, so far, two HDAC inhibitors are approved by the US Food and Drug Administration (FDA)."

Compared to HDAC inhibitors, the development of HMT inhibitors is much less advanced, but growing evidence implicates several HMTs as important drug targets in various cancers, says Yongcheng Song, Assistant Professor at Baylor College of Medicine, Texas, USA. "One example is DOT1L, an HMT that specifically methylates histone 3 lysine 79 (H3K79), which is a target for acute leukaemias with translocations of the MLL gene. Several predominant fusion partners of onco-MLL have been shown to recruit DOT1L, and $\mathrm{H} 3 \mathrm{~K} 79$ hypermethylation was also found to be a hallmark of MLL-rearranged leukaemias."

Epizyme's DOT1L inhibitor EPZ004777 has been shown to selectively inhibit the proliferation of MLL-rearranged leukaemia cell lines and prolong survival in a mouse xenograft model of MLL (Cancer Cell 20, 53-65; 2011). However, further development is needed before DOT1L inhibitors can become clinically useful drugs, says Song, in particular to improve on the poor pharmacokinetic properties of EPZ004777, which has a very short plasma half-life. "Combination therapy should also be applied to further develop such compounds" he adds. "The rationale is that non-cytotoxic DOT1L inhibitors, which induce differentiation and apoptosis of leukaemic stem cells (or leukaemia-initiating cells), work slowly on this aggressive disease, and so combination with a cytotoxic drug that quickly kills non-stem leukaemia cells, thereby reducing overall cancer burden, should be useful." Chen also notes that DOT1L is widely expressed in human tissues and likely to be involved in several biological processes, raising questions about the potential toxicity of DOT1L inhibitors.

With regard to targeting HMTs in general, a major challenge is how to find selective inhibitors, given that more than 50 human HMTs use $\mathrm{S}$-adenosylmethionine (SAM) as a common cofactor, says Song. However, the development of EPZ004777 and several other selective DOT1L inhibitors that target the SAM-binding site has indicated that ligand-based medicinal chemistry, guided by structure-based design, can be a viable approach for the discovery of potent and selective HMT inhibitors, he adds. Given that multiple companies are now developing such inhibitors, Chen concludes that targeting HMTs, as well as other emerging epigenetic modifiers such as lysine demethylases, will be a promising new frontier for anticancer drug discovery.

Alexandra Flemming 